\title{
On $q$-poly-Bernoulli numbers arising from combinatorial interpretations
}

\author{
BeÁtA BÉnyi(iD AND José L. Ramírez
}

\begin{abstract}
In this paper we present several natural $q$-analogues of the poly-Bernoulli numbers arising in combinatorial contexts. We also recall some related analytical results and ask for combinatorial interpretations.
\end{abstract}

Mathematics Subject Classification. 05A05, 05A19.

Keywords. Poly-Bernoulli number, $q$-analogue, Combinatorial interpretation.

\section{Introduction}

Poly-Bernoulli numbers were introduced by Kaneko in 1997 [20] as he noticed that the generating function of the usual Bernoulli numbers can be generalized in a nice way using the polylogarithm function. Later, in 1999 Arakawa and Kaneko [2] observed that poly-Bernoulli numbers can be expressed as special values of multiple zeta values. Motivated by this observation, they introduced a function, nowadays known as the Arakawa-Kaneko function, which expresses special values of this function at negative integers with the help of poly-Bernoulli numbers and multiple zeta values. Since then poly-Bernoulli numbers have been studied by numerous authors.

The literature in this topic is so rich that we cannot give here a complete list of all the areas that have been motivated by these works, however, we mention a few. Generalizations were introduced, as for instance multi-polyBernoulli numbers [5] or poly-Bernoulli numbers associated with a Dirichlet character [4]. Analogue numbers were also introduced as poly-Euler [31], polyCauchy [22], poly-Eulerian numbers [34]. Beyond the numbers, polynomials (and generalizations of the polynomials) were defined and studied (see for instance $[23,26])$. In another direction, using the formula of poly-Bernoulli numbers, that involves the Stirling numbers of the second kind, the authors 
replaced in the formula variations of the Stirling numbers and studied the so obtained number sequences and polynomials, respectively. For example, the classical Stirling numbers are replaced by the incomplete Stirling numbers $[11,24]$ or the $r$-Stirling numbers $[10,25]$.

Poly-Bernoulli numbers received attention from combinatorialists because poly-Bernoulli numbers with negative $k$ indices enumerate various combinatorial objects and have very interesting combinatorial properties. We find also generalizations in the literature that are natural from the combinatorial point of view. One of the first combinatorial interpretations was given by Brewbaker [12] in terms of lonesum matrices.

In combinatorics, number theory, and the theory of special polynomials the so called $q$-analogues of number sequences are often investigated. In combinatorial context $q$ is usually used for a parameter of the underlying combinatorial object which is enumerated by the number itself if the extra parameter is not taking into account (so for $q=1$ ). In this paper we focus on the aspects of $q$-analogues of the poly-Bernoulli numbers. There are different ways to attack this problem. The aim of this paper is to show some of these directions. Most of all we want to highlight the combinatorial richness of the theory of polyBernoulli numbers by defining several $q$-analogues of this counting sequence that arise naturally from the combinatorial interpretations. We also point out a $q$-analogue which was defined analytically and give an appropriate combinatorial interpretation by using colored lonesum matrices. We illustrated our results with examples in order to facilitate the reading of the paper and to show the wealth of the theory.

The outline of the paper is as follows. First, we recall some necessary definitions and notation we use throughout the paper. The definitions that are specific for the considered $q$-analogue in a certain section is given in the section itself. In Sect. 3, we give a natural combinatorial interpretation of the $q$-poly-Bernoulli numbers by using ordered partitions and inversion statistics. In Sect. 4, we use the weight defined by Cigler in his study. We interpret this weight on the set of lonesum matrices to give a new $q$-analogue. In Sect. 5, we exploit the interpretation of poly-Bernoulli numbers in the context of Vesztergombi permutations. Then, by using the $q$-rook numbers studied by Garsia and Remmel, we give a combinatorial identity to find these new polynomials. In Sect. 6, we show some relations between the $q$-analogue defined by Cenkci and Komatsu and the $\Gamma$-free matrices. Additionally, we introduce the colored lonesum matrices.

\section{Definition and notation}

Poly-Bernoulli numbers, denoted by $B_{n}^{(k)}$, where $n$ is a positive integer and $k$ is an integer, are defined by the following exponential generating function [20] 


$$
\sum_{n=0}^{\infty} B_{n}^{(k)} \frac{x^{n}}{n !}=\frac{\operatorname{Li}_{k}\left(1-e^{-x}\right)}{1-e^{-x}},
$$

where $\operatorname{Li}_{k}(z)=\sum_{i=1}^{\infty} z^{i} / i^{k}$ is the $k$-th polylogarithm function. Note that for $k=1$ we recover the classical Bernoulli numbers, that is, $B_{n}^{(1)}=(-1)^{n} B_{n}$, for $n \geq 0$, where $B_{n}$ denotes the $n$-th Bernoulli number.

From the combinatorial point of view the array with negative $k$ indices are interesting, since these numbers are integers (see sequence A099594 in [33]). The first few values of this array are

\begin{tabular}{c|cccccc}
$k \backslash n$ & 0 & 1 & 2 & 3 & 4 & 5 \\
\hline 0 & 1 & 1 & 1 & 1 & 1 & 1 \\
-1 & 1 & 2 & 4 & 8 & 16 & 32 \\
-2 & 1 & 4 & 14 & 46 & 146 & 454 \\
-3 & 1 & 8 & 46 & 230 & 1066 & 4718 \\
-4 & 1 & 16 & 146 & 1066 & 6902 & 41506 \\
-5 & 1 & 32 & 454 & 4718 & 41506 & 329462
\end{tabular}

Several explicit formulas and recurrence relations for the poly-Bernoulli numbers are known:

$$
\begin{gathered}
B_{n}^{(k)}=(-1)^{n} \sum_{m=0}^{n}(-1)^{m} \frac{m !\left\{\begin{array}{c}
n \\
m
\end{array}\right\}}{(m+1)^{k}}, \\
B_{n}^{(-k)}=\sum_{m=0}^{\min (n, k)} m !\left\{\begin{array}{c}
n+1 \\
m+1
\end{array}\right\} m !\left\{\begin{array}{c}
k+1 \\
m+1
\end{array}\right\}, \\
B_{n}^{(-k-1)}=B_{n}^{(-k)}+\sum_{m=1}^{n}\left(\begin{array}{c}
n \\
m
\end{array}\right) B_{n-(m-1)}^{(-k)}
\end{gathered}
$$

The explicit formula (2) was first presented by Kaneko in [20] and the closed formula (3) in [3]. Both formulas were proven combinatorially first in [12]. The recurrence (4) was shown by Kaneko [21] and combinatorially in [7].

In the formulas (2) and (3) we see that Stirling numbers of the second kind play a key role. Stirling numbers of the second kind, $\left\{\begin{array}{l}n \\ k\end{array}\right\}$, are defined as the number of set partitions of an $n$-element set into $k$ non-empty blocks. Stirling numbers of the second kind have several $q$-analogues introduced in the literature (cf. $[29,30])$. In this paper we use different definitions, appropriate for our purposes of the specific sections.

The first combinatorial interpretation of poly-Bernoulli numbers are lonesum matrices [12]. Lonesum matrices are binary matrices that are uniquely 
reconstructible from their column and row sum vectors. They can be characterized by forbiddance of submatrices of the form

$$
\left(\begin{array}{ll}
0 & 1 \\
1 & 0
\end{array}\right) \text { or }\left(\begin{array}{ll}
1 & 0 \\
0 & 1
\end{array}\right) \text {. }
$$

Similarly, there are three other pairs of $2 \times 2$ submatrices such that their forbiddance leads to a class of $(0,1)$-matrices enumerated by the poly-Bernoulli numbers, see for example $[7,8]$. The formula (3) enumerates pairs of ordered partitions, which is an obvious interpretation and can be turned immediately to a so called Callan permutation [7]. Another permutation class, which we call Vesztergombi permutations, was found early in an algebraic study [28]. In this paper we will only refer to these interpretations though there are also graph theoretical applications (cf. [10]), for instance.

We recall the particular $q$-analogue in the section itself. They involve the $q$-numbers as explicitly used by Jackson [19], see also [1, section 10.2.3]. Let $q$ be a variable satisfying $0<|q|<1$. For complex $x$ the $q$-number of $x$ is defined to be $[x]_{q}:=\left(1-q^{x}\right) /(1-q)$. For $n$ a positive integer we have

$$
[n]_{q}:=\frac{1-q^{n}}{1-q}=1+q+q^{2}+\cdots+q^{n-1} .
$$

We also use the notation of the $q$-factorial $[n] !_{q}:=[1]_{q}[2]_{q} \cdots[n]_{q}$. If it is not confusing and not necessary to write explicitly out we drop the subscript $q$ and simply write $[n]$ instead of $[n]_{q}$. Similarly, $[n]$ ! instead of $[n] !_{q}$. Note that the above expressions become the integer $n$ and the factorial $n$ ! when $q=1$.

\section{3. $q$-analogue based on the obvious interpretation}

First, we generalize the formula (3) in a straightforward manner. Carlitz [14, Eq. (3.2)] introduced a $q$-Stirling number that satisfies the following recursion:

$$
\left\{\begin{array}{l}
n \\
m
\end{array}\right\}_{q}=\left\{\begin{array}{c}
n-1 \\
m-1
\end{array}\right\}_{q}+[m]_{q}\left\{\begin{array}{c}
n-1 \\
m
\end{array}\right\}_{q},
$$

with the initial condition $\left\{\begin{array}{c}n \\ 0\end{array}\right\}_{q}=\left\{\begin{array}{l}0 \\ m\end{array}\right\}_{q}=0$ except $\left\{\begin{array}{l}0 \\ 0\end{array}\right\}_{q}=1$. Notice that this is only one of the definitions given by Carlitz for the $q$-Stirling numbers. Combinatorial interpretations are given for instance in [29].

Definition 1. We define the following $q$-analogue of poly-Bernoulli numbers for nonnegative integers $n, k$ and a real number $q$ with $q \neq 0$ :

$$
B_{n, q}^{(k)}:=\sum_{m=0}^{\min (n, k)}[m] !\left\{\begin{array}{l}
n+1 \\
m+1
\end{array}\right\}_{q}[m] !\left\{\begin{array}{c}
k+1 \\
m+1
\end{array}\right\}_{q} .
$$


Before we give the combinatorial interpretation of these numbers it is useful to consider the $q$-analogue of the Fubini numbers.

Fubini numbers, also called ordered Bell numbers, denoted by $F_{n}$, count the total number of set partitions of $\{1,2, \ldots, n\}$ where the blocks are ordered. They are given by

$$
F_{n}=\sum_{k=0}^{n} k !\left\{\begin{array}{l}
n \\
k
\end{array}\right\} \text { for } n>0, \text { and } F_{0}=1 .
$$

Note that the combinatorial identity (3) of poly-Bernoulli numbers has a great similarity with (7) of Fubini numbers. For this reason, in this section we will study a $q$-analogue of the Fubini numbers. We present a combinatorial interpretation by using inversions.

Definition 2. [29, p. 205] Let $\omega=B_{1} / B_{2} / \cdots / B_{k}$ be a set partition, and $b \in$ $B_{i}$. The pair $\left(b, B_{j}\right)$ is an inversion if $b>\min B_{j}$ and $i<j$. The inversion number of $\omega$, written $\operatorname{inv}^{*}(\omega)$, is the number of inversions in $\omega$.

For example, if $\omega=137 / 26 / 45$ then $\left(3, B_{2}\right),\left(7, B_{2}\right),\left(7, B_{3}\right)$, and $\left(6, B_{3}\right)$ are the inversions of $\omega$, and $\operatorname{inv}^{*}(\omega)=4$. Note that the $q$-Stirling numbers defined by the recursion (5) have the following combinatorial interpretation (cf. [29, p. 205])

$$
\left\{\begin{array}{l}
n \\
k
\end{array}\right\}_{q}=\sum_{\omega \in \Pi_{n, k}} q^{\operatorname{inv}^{*}(\omega)},
$$

where $\Pi_{n, k}$ is the set of partitions of $\{1,2, \ldots, n\}$ into $k$ blocks.

We define the $q$-Fubini numbers using $q$-analogues of the expressions of (7) by the equality

$$
F_{n, q}:=\sum_{k=0}^{n}[k]_{q} !\left\{\begin{array}{l}
n \\
k
\end{array}\right\}_{q} .
$$

It is clear that $\lim _{q \rightarrow 1} F_{n, q}=F_{n}$. The first few $q$-Fubini numbers are

$1, \quad 1, \quad q+2, \quad q^{3}+3 q^{2}+5 q+4, \quad q^{6}+4 q^{5}+9 q^{4}+16 q^{3}+20 q^{2}+17 q+8, \ldots$

Theorem 3. The q-Fubini numbers are given by

$$
F_{n, q}=\sum_{\pi \in O_{n}} q^{\operatorname{inv}^{*}(\pi)},
$$

where $O_{n}$ is the set of ordered set partitions of $\{1,2, \ldots, n\}$.

Proof. Let $T_{q}(n, k):=[k]_{q} !\left\{\begin{array}{l}n \\ k\end{array}\right\}_{q}$. From (5) it is possible to prove that $T_{q}(n, k)$ satisfies the recurrence relation

$$
T_{q}(n, k)=[k]_{q}\left(T_{q}(n-1, k-1)+T_{q}(n-1, k)\right),
$$


with the initial condition $T_{q}(n, 0)=0=T_{q}(0, k)$ for $n>0$ and $T_{q}(0,0)=1$. On the other hand, let $U_{q}(n, k):=\sum_{\pi \in O_{n, k}} q^{\text {inv }^{*}(\pi)}$, where $O_{n, k}$ is the set of ordered set partitions of $\{1,2, \ldots, n\}$ into $k$ non-empty blocks. The sequence $U_{q}(n, k)$ satisfies the same recurrence relation of $T_{q}(n, k)$, with the same initial values. Indeed, for any ordered partition $\pi$ of $\{1,2, \ldots, n\}$ into $k$ non-empty blocks, there are two options: either the element $n$ is in a single block or it is in a block with more than one element. In the first case, if the single block is in the $i$-th position, the contribution is $q^{k-i} U_{q}(n-1, k-1)$, for $i=1,2, \ldots, k$. Summing over the possible values of $i$, we obtain $[k]_{q} U_{q}(n-1, k-1)$.

In the second case, the element $n$ can be placed into one of the $k$ blocks. Then its contribution is $q^{k-i} U_{q}(n-1, k)$, where $i$ is the position of the block from left to right. Summing over the possible values of $i$, we obtain $[k]_{q} U_{q}(n-$ $1, k)$. Therefore, we conclude that $U_{q}(n, k)=T_{q}(n, k)$ for all $n, k \geq 0$.

For example, $F_{3, q}=q^{3}+3 q^{2}+5 q+4$, with the ordered partitions and their respective weights being

$$
\begin{array}{llll}
\{1,2,3\} \rightarrow 1 & \{1,2\},\{3\} \rightarrow 1 & \{3\},\{1,2\} \rightarrow q & \{1,3\},\{2\} \rightarrow q \\
\{2\},\{1,3\} \rightarrow q & \{2,3\},\{1\} \rightarrow q^{2} & \{1\},\{2,3\} \rightarrow 1 & \{1\},\{2\},\{3\} \rightarrow 1 \\
\{1\},\{3\},\{2\} \rightarrow q & \{2\},\{1\},\{3\} \rightarrow q & \{2\},\{3\},\{1\} \rightarrow q^{2} & \{3\},\{2\},\{1\} \rightarrow q^{3} \\
\{3\},\{1\},\{2\} \rightarrow q^{2} & & &
\end{array}
$$

Now we turn our attention to the combinatorial interpretation of the formula (6).

The formula (3) has the following obvious interpretation. Let $\widehat{N}$ denote the set $\{1,2, \ldots, n\}$ extended by a special element $\overline{0}$ and $\widehat{K}$ the set $\{1,2, \ldots, k\}$ extended by another special element $\overline{k+1}$. Equation (3) counts the alternating sequence of blocks of partitions of $\widehat{N}$ and $\widehat{K}$ such that the first block contains $\overline{0}$ and the last block contains $\overline{k+1}$ [7]. Note that the number of blocks of $\widehat{N}$ and $\widehat{K}$ has to be equal to provide such an alternating sequence. The interpretation of the $q$-analogue of poly-Bernoulli numbers $B_{n, q}^{(k)}$ should be clear based on this fact and the interpretation of $q$-Fubini numbers. We formulate it precisely in the next theorem.

Theorem 4. Let $\widehat{N}=\{\overline{0}, 1, \ldots, n\}$ and $\widehat{K}=\{1,2, \ldots, k, \overline{k+1}\}$ be two sets. Let $\pi_{n, k}$ denote an alternating sequence of blocks of partitions of $\widehat{N}$ and $\widehat{K}$ such that the first block contains $\overline{0}$ and the last block contains $\overline{k+1}$. Further, let $\mathcal{O} \mathcal{P}_{n, k}$ denote the set of all such alternating sequences. The q-poly-Bernoulli numbers $B_{n, q}^{(k)}$ count the inversions in the sequences $\pi_{n, k}$ :

$$
B_{n, q}^{(k)}=\sum_{\pi_{n, k} \in \mathcal{O P}_{n, k}} q^{\mathrm{inv}^{*}\left(\pi_{n, k}\right)} .
$$


For example, $B_{3, q}^{(1)}=4+3 q+q^{2}$. This polynomial can be obtained by counting the inversions in the pairs of ordered partitions of the augmented sets $\{\overline{\mathbf{0}}, \mathbf{1}, \mathbf{2}, \mathbf{3}\}$ and $\{1, \overline{2}\}$. We list the elements of $\mathcal{O} \mathcal{P}_{3,1}$ with their weights.

$$
\begin{array}{lccc}
\overline{\mathbf{0}} \mathbf{1 2 3} / 1 \overline{2} \rightarrow 1 & \overline{\mathbf{0}} / 1 / \mathbf{1 2 3} / \overline{2} \rightarrow 1 & \overline{\mathbf{0}} \mathbf{1} / 1 / \mathbf{2 3} / \overline{2} \rightarrow 1 & \overline{\mathbf{0} 23} / 1 / \mathbf{1} / \overline{2} \rightarrow q^{2} \\
\overline{\mathbf{0}} \mathbf{1 2} / 1 / \mathbf{3} / \overline{2} \rightarrow 1 & \overline{\mathbf{0}} \mathbf{3} / 1 / \mathbf{1 2} / \overline{2} \rightarrow q & \overline{\mathbf{0}} \mathbf{1 3} / 1 / \mathbf{2} / \overline{2} \rightarrow q & \overline{\mathbf{0} 2} / 1 / \mathbf{1 3} / \overline{2} \rightarrow q
\end{array}
$$

Notice that the number of blocks of the partitions of the two sets $\widehat{N}$ and $\widehat{K}$ is equal.

There are some known formulas for Carlitz's $q$-Stirling numbers. These combinatorial formulas allow us to calculate the $q$-analogue of the poly-Bernoulli numbers $B_{n, q}^{(k)}$ in terms of $q$-binomial coefficients. Remember that the $q$-binomial coefficient is defined by (cf. [1, p. 483])

$$
\left[\begin{array}{l}
n \\
k
\end{array}\right]_{q}=\frac{[n] !}{[k] ![n-k] !} .
$$

Moreover, these coefficients satisfy the recurrence relation

$$
\left[\begin{array}{l}
n \\
k
\end{array}\right]_{q}=\left[\begin{array}{l}
n-1 \\
k-1
\end{array}\right]_{q}+q^{k}\left[\begin{array}{c}
n-1 \\
k
\end{array}\right]_{q} .
$$

Theorem 5. The polynomials $B_{n, q}^{(k)}$ are given by

$$
\begin{aligned}
B_{n, q}^{(k)}= & \sum_{m=0}^{\min \{n, k\}} \frac{1}{[m+1]^{2}} \sum_{i=0}^{2 m+2} \\
& \sum_{\ell=0}^{i}(-1)^{i} q^{\left(\begin{array}{c}
m+1-\ell \\
2
\end{array}\right)+\left(\begin{array}{c}
m+1-i+\ell \\
2
\end{array}\right)-2\left(\begin{array}{c}
m+1 \\
2
\end{array}\right)}\left[\begin{array}{c}
m+1 \\
\ell
\end{array}\right]_{q}\left[\begin{array}{c}
m+1 \\
i-\ell
\end{array}\right]_{q}[\ell]^{n+1}[i-\ell]^{k+1} .
\end{aligned}
$$

Proof. Carlitz [14, Eq. (3.3)] proved that the $q$-Stirling numbers of the second kind are given by the expression

$$
\left\{\begin{array}{c}
n \\
m
\end{array}\right\}_{q}=\frac{1}{[m] !} \sum_{i=0}^{m}(-1)^{m-i} q^{\left(\begin{array}{c}
m-i \\
2
\end{array}\right)-\left(\begin{array}{c}
m \\
2
\end{array}\right)}\left[\begin{array}{c}
m \\
i
\end{array}\right]_{q}[i]^{n} .
$$

From the above equation and Cauchy's product we obtain the desired result.

From Theorem 5 we obtain that the first few polynomials $B_{n, q}^{(1)}$ are

$$
\begin{aligned}
& B_{0, q}^{(1)}=1, \\
& B_{1, q}^{(1)}=2, \\
& B_{2, q}^{(1)}=q+3, \\
& B_{3, q}^{(1)}=q^{2}+3 q+4, \\
& B_{4, q}^{(1)}=q^{3}+4 q^{2}+6 q+5, \\
& B_{5, q}^{(1)}=q^{4}+5 q^{3}+10 q^{2}+10 q+6,
\end{aligned}
$$




$$
\begin{aligned}
& B_{6, q}^{(1)}=q^{5}+6 q^{4}+15 q^{3}+20 q^{2}+15 q+7, \\
& B_{7, q}^{(1)}=q^{6}+7 q^{5}+21 q^{4}+35 q^{3}+35 q^{2}+21 q+8 .
\end{aligned}
$$

The coefficients of the above polynomials have an interesting combinatorial meaning (see array A299927 in [33]). Let $a(n, m)$ be the number of permutations of length $n$ that avoid the patterns 213 and 312, and have $m$ double ascents. Let $\pi$ be a permutation on a set of $n$ elements. The double ascent dasc is a permutation statistic defined by

$$
\operatorname{dasc}(\pi):=\mid\left\{i: \pi_{i}<\pi_{i+1} \text { and } \pi_{i+1}<\pi_{i+2}\right\} \mid .
$$

For example, $a(5,2)=4$, the relevant permutations being

$$
\text { 12354, 12453, 13452, } 23451 .
$$

Bukata et al. [13] proved that

$$
a(n, m)= \begin{cases}n, & \text { if } m=0 \\
\left(\begin{array}{l}
n-1 \\
m+1
\end{array}\right), & \text { if } m \geq 1\end{cases}
$$

Theorem 6. For $n \geq 0$, we have

$$
B_{n, q}^{(1)}=\sum_{m=0}^{n-1} a(n+1, m) q^{m} .
$$

Proof. The case $n=0$ is clear. Suppose that $n \geq 1$. From Definition (6) we have

$$
B_{n, q}^{(1)}=\left\{\begin{array}{c}
n+1 \\
1
\end{array}\right\}_{q}\left\{\begin{array}{l}
2 \\
1
\end{array}\right\}_{q}+\left\{\begin{array}{c}
n+1 \\
2
\end{array}\right\}_{q}\left\{\begin{array}{l}
2 \\
2
\end{array}\right\}_{q}=\left\{\begin{array}{c}
n+1 \\
2
\end{array}\right\}_{q}+1 .
$$

Recall that $\left\{\begin{array}{c}n+1 \\ 2\end{array}\right\}_{q}=\sum_{\omega \in \Pi_{n+1,2}} q^{\text {inv* }^{*}(\omega)}$. The coefficient of $q$ in the sum is the number of set partitions of $\{1, \ldots, n+1\}$ into two blocks with $m$ inversions, i.e., with $m$ pairs $\left(b, B_{j}\right)$, such that $b \in B_{i}$ with $b<\min B_{j}$ and $i<j$. In this special case this means that we partition $n+1$ elements into two blocks, so that the block with the smallest least element (which is necessarily 1) contains $m$ elements that are greater than the least element of the other block. Such a partition is easy to construct: arrange the elements and select exactly $m+1$ elements from $\{2,3, \ldots, n+1\}$. The least selected element indicates the least element of the second block (since this has to be the block with the greater least element, 1 can not be selected). All the elements to the left of this element will be contained in the first block, while the elements to the right will be contained in the second block, except those that are also selected, namely these selected elements will be also contained in the first block - and these form the $m$ pairs of inversions with the least selected element, the least element of the second block. This gives $\left(\begin{array}{c}n \\ m+1\end{array}\right)$ cases, which is $a(n+1, m)$ as desired if $m \neq 0$. If 
$m=0$, we have $n$ choices, so the constant term in $\left\{\begin{array}{c}n+1 \\ 2\end{array}\right\}_{q}$ is $n$, and hence the constant term in $B_{n, q}^{(1)}=\left\{\begin{array}{c}n+1 \\ 2\end{array}\right\}_{q}+1$ is $n+1$, which is $a(n+1,0)$.

\section{4. q-analogue on lonesum matrices}

In this section we introduce another $q$-analogue that arises naturally as a generalization of the formula (3). For this aim we recall a $q$-analogue of the Stirling numbers of the second kind defined by Cigler [16] based on a block of a set partition that contains a particular element.

Let us denote by $\Pi(n, k)$ the set of partitions of $\{0,1, \ldots, n-1\}$ into $k$ non-empty blocks. It is clear that $|\Pi(n, k)|=\left\{\begin{array}{l}n \\ k\end{array}\right\}$. Suppose $\pi \in \Pi(n, k)$ is represented as $B_{0} / B_{1} / \cdots / B_{k-1}$, where $B_{0}$ denotes the block containing the element zero. Define the weight $w_{1}$ of the partition $\pi$ by letting $w_{1}(\pi):=$ $q^{\sum_{i \in B_{0}}{ }^{i}}$.

The $q$-Stirling numbers of the second kind [16], denoted by $\left\{\begin{array}{l}n \\ k\end{array}\right\}_{q}^{*}$, are defined by

$$
\left\{\begin{array}{l}
n \\
k
\end{array}\right\}_{q}^{*}=\sum_{\pi \in \Pi(n, k)} q^{w_{1}(\pi)} .
$$

This sequence can be also defined as the connecting coefficients in the expression

$$
(x+1)(x+2) \cdots\left(x+q^{n-1}\right)=\sum_{k=1}^{n}\left\{\begin{array}{l}
n \\
k
\end{array}\right\}_{q}^{*}(x+1)^{\underline{k}},
$$

where $x^{\underline{n}}:=x(x-1) \cdots(x-n+1)$ for $n \geq 1$, and $x^{\underline{0}}=1$. Moreover, this sequence satisfies the recurrence relation

$$
\left\{\begin{array}{l}
n \\
m
\end{array}\right\}_{q}^{*}=\left\{\begin{array}{c}
n-1 \\
m-1
\end{array}\right\}_{q}^{*}+\left(m-1+q^{n-1}\right)\left\{\begin{array}{c}
n-1 \\
m
\end{array}\right\}_{q}^{*}
$$

with the initial condition $\left\{\begin{array}{c}n \\ 0\end{array}\right\}_{q}^{*}=\left\{\begin{array}{c}0 \\ m\end{array}\right\}_{q}^{*}=0$, except $\left\{\begin{array}{l}0 \\ 0\end{array}\right\}_{q}^{*}=1$.

Based on this definition, we introduce the following $q$-analogue of polyBernoulli numbers. As we mentioned in the introduction a 01 matrix is called lonesum if it is uniquely reconstructible from its row and column sum vectors.

For example, $B_{2}^{(-3)}=46$, the lonesum matrices of size $2 \times 3$ appear in Fig. 1. Note that we use black squares for the ones and white squares for the zeros.

Let $\mathcal{L}(n, k)$ be the set of lonesum matrices of size $n \times k$. Brewbaker [12] proved that

$$
|\mathcal{L}(n, k)|=\sum_{m=0}^{\min \{n, k\}} m !\left\{\begin{array}{c}
n+1 \\
m+1
\end{array}\right\} m !\left\{\begin{array}{c}
k+1 \\
m+1
\end{array}\right\}
$$



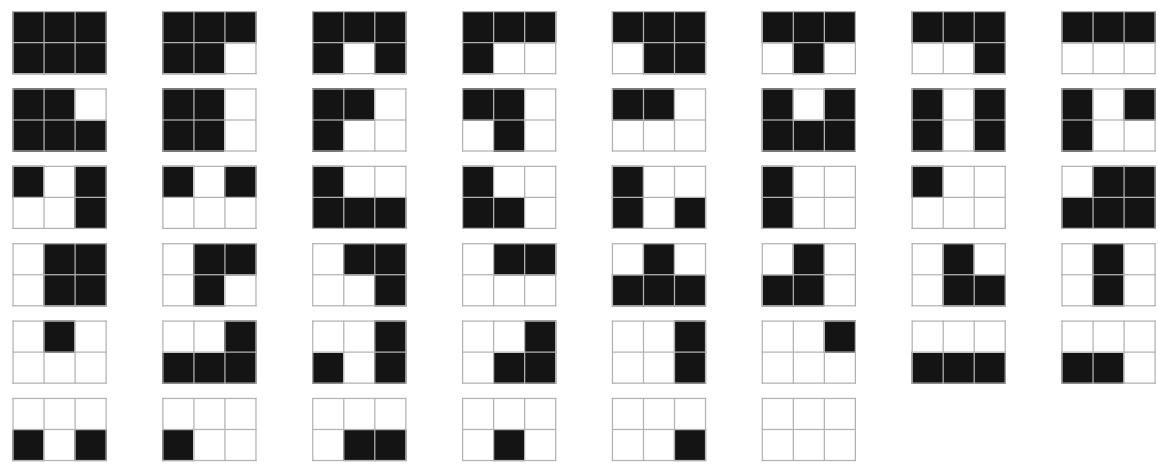

Figure 1. Lonesum matrices of size $2 \times 3$

Definition 7. We define the following $q$-analogue of the poly-Bernoulli numbers:

$$
p_{q}(n, k):=\sum_{m=0}^{\min \{n, k\}} m !\left\{\begin{array}{c}
n+1 \\
m+1
\end{array}\right\}_{q}^{*} m !\left\{\begin{array}{c}
k+1 \\
m+1
\end{array}\right\}_{q}^{*} .
$$

Given a matrix $A$, we define the set $\mathcal{N}(A)$ as the indices of the all-0 columns and all-0 rows of $A$. The weight $w_{2}$ of a lonesum matrix $A$ is defined by letting $w_{2}(A):=q^{\sum_{i \in \mathcal{N}}{ }^{i}}$.

For example, for the matrix

$$
A=\left(\begin{array}{lllllllll}
1 & 1 & 1 & 0 & 0 & 1 & 1 & 1 & 0 \\
1 & 0 & 1 & 0 & 0 & 1 & 0 & 1 & 0 \\
0 & 0 & 0 & 0 & 0 & 0 & 0 & 0 & 0 \\
1 & 1 & 1 & 1 & 0 & 1 & 1 & 1 & 0 \\
1 & 0 & 1 & 0 & 0 & 1 & 0 & 1 & 0 \\
1 & 1 & 1 & 0 & 0 & 1 & 1 & 1 & 0
\end{array}\right)
$$

we have that $\mathcal{N}(A)=\{3,5,9\}$. Therefore $w_{2}(A)=q^{17}$.

Theorem 8. We have

$$
p_{q}(n, k)=\sum_{A \in \mathcal{L}(n, k)} q^{w_{2}(A)}=\sum_{m=0}^{\min \{n, k\}} m !\left\{\begin{array}{c}
n+1 \\
m+1
\end{array}\right\}_{q}^{*} m !\left\{\begin{array}{c}
k+1 \\
m+1
\end{array}\right\}_{q}^{*} .
$$

Proof. A lonesum matrix $A$ is determined by a pair of ordered partitions. See a detailed proof of how to construct bijectively a lonesum matrix from a given ordered partition pair for instance $[6-8,12]$. The key observation in a lonesum matrix is that two rows $R$ and $R^{\prime}$ are either identical or the one with more 1 entries, say $R^{\prime}$, can be obtained from $R$ by switching some $0 \mathrm{~s}$ in $R$ to 1 s. The same is true for the columns. Hence, an ordered partition of the row indices corresponds to the order of the rows as follows: the indices of the identical rows 
are contained in the same block, and the order is determined by the number of $1 \mathrm{~s}$ in the particular rows. For the special all-zero rows, we introduce a special block, by adding 0 to the set of the row-indices and taking the ordered partition of the extended set. Similarly, for columns. According to this procedure, the block containing 0 , contains the row indices of the all-zero rows. (The same is true for columns.) The theorem follows.

Theorem 9. The polynomials $p_{q}(n, k)$ are given by the expression

$$
\begin{aligned}
p_{q}(n, k)= & \sum_{m=0}^{\min \{n, k\}} \frac{1}{(m+1)^{2}} \sum_{j=0}^{2 m+2} \sum_{\ell=0}^{j}(-1)^{j}\left(\begin{array}{c}
m+1 \\
\ell
\end{array}\right)\left(\begin{array}{c}
m+1 \\
j-\ell
\end{array}\right) \\
& \times\left(\prod_{i=0}^{n}\left(q^{i}+\ell-1\right)\right)\left(\prod_{i=0}^{k}\left(q^{i}+j-\ell-1\right)\right) .
\end{aligned}
$$

Proof. Corcino and Fernandez [17] proved that the $q$-Stirling numbers of Cigler are given by the combinatorial formula

$$
\left\{\begin{array}{c}
n \\
m
\end{array}\right\}_{q}^{*}=\frac{1}{m !} \sum_{i=0}^{m}(-1)^{m-i}\left(\begin{array}{c}
m \\
i
\end{array}\right) \prod_{j=0}^{n-1}\left(q^{j}+i-1\right) .
$$

From the above equation and Cauchy's product we obtain the desired result.

\section{Inversions in Vesztergombi permutations}

In this section we describe a natural $q$-analogue of poly-Bernoulli numbers based on a permutation class. Vesztergombi [35] investigated permutations with restrictions on the distance of the elements and their images (considering a permutation as a bijection on $\{1,2, \ldots, n\})$. Launois [28] noticed that the number of such permutations with a particular bound is given by a polyBernoulli number. Several combinatorial proofs were later found for this fact [6-8]. The $q$-analogue we introduce now arose in [32] during the study of intervals in Bruhat order. Here, we focus on the pure combinatorial point of view and on the theory of poly-Bernoulli numbers.

Definition 10. A permutation $\pi$ of $[n+k]$ is called Vesztergombi permutation if

$$
-k \leq \pi(i)-i \leq n,
$$

for all $i \in[n+k]$.

Theorem 11. ([28]) Let $\mathcal{V}_{n}^{k}$ denote the set of Vesztergombi permutations. Then

$$
\left|\mathcal{V}_{n}^{k}\right|=B_{n, k} .
$$



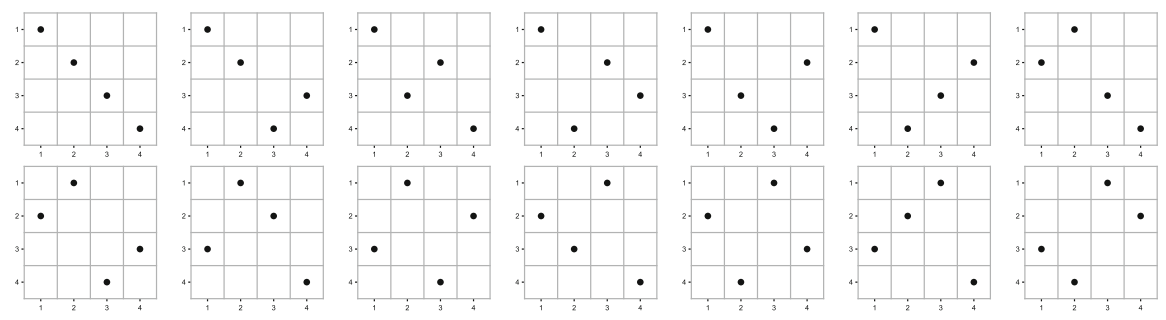

FIGURE 2. Vesztergombi permutations of size $[2+2]$

For example, from the permutations in Fig. 2 we have $B_{2}^{(-2)}=14$. Note that for a permutation $\pi \in \mathcal{V}_{n}^{k}$ this array is obtained by placing a dot in row $i$ and column $\pi_{i}$ of an $(n+k) \times(n+k)$ array.

Various proofs can be found in the literature: analytical [35], by constructing a bijection between lonesum matrices and Vesztergombi permutations [27], or using graph theoretical methods [6]. Here, we will use the description of the proof in [8], since this is the one that can be used in the $q$-generalization. For a permutation $\pi$, we denote by $\operatorname{inv}(\pi)$ the number of inversions of $\pi$, i.e., the number of pairs $(i, j), i<j$ such that $\pi_{i}>\pi_{j}$.

Definition 12. We define the $q$-analogue $p B_{n, k}(q)$ as follows:

$$
p B_{n, k}(q):=\sum_{\pi \in \mathcal{V}_{n}^{k}} q^{\operatorname{inv}(\pi)} .
$$

For example, from permutations in Fig. 2 we have

$$
p B_{2,2}(q)=1+3 q+5 q^{2}+4 q^{3}+q^{4} .
$$

In Theorem 13 we show a combinatorial identity for the sequence $p B_{n, k}(q)$. Here we use the $q$-Stirling numbers $S_{n, k}(q)$ defined by the following recurrence (cf. [29, p. 168])

$$
S_{n+1, k}(q)=q^{k-1} S_{n, k-1}+[k]_{q} S_{n, k}(q), \quad \text { for } 0 \leq k \leq n,
$$

with the initial conditions $S_{0,0}(q)=1$ and $S_{n, k}(q)=0$ for $k<0$ or $k>n$.

Notice that these $q$-Stirling numbers have the following combinatorial interpretation

$$
S_{n, k}(q)=\sum_{\pi \in \Pi_{n, k}} q^{\operatorname{inv}^{*}(\pi)+\left(\begin{array}{l}
k \\
2
\end{array}\right)} .
$$

Theorem 13. We have the combinatorial identity

$$
p B_{n, k}(q)=q^{n k} \sum_{m=0}^{\min (n, k)} S_{n+1, m+1}(1 / q) S_{k+1, m+1}(1 / q)[m] !_{q}^{2} q^{m} .
$$


One geometrical visualization of a Vesztergombi permutation can be given on a $(n+k) \times(n+k)$ matrix $V_{n+k}$ defined by:

$$
v_{i j}=\left\{\begin{array}{l}
1, \text { if }-k \leq i-j \leq n, \quad i, j=1, \ldots, n+k \\
0, \text { otherwise }
\end{array}\right.
$$

The matrix $V$ is consists of 4 blocks, two all-1 matrices $\left(J_{n, k}, J_{k, n}\right)$, a lower $\left(T_{n}\right)$ and an upper $\left(T^{k}\right)$ triangular matrix. Precisely, let $V_{n+k}$ be the matrix:

$$
V_{n+k}=\left(\begin{array}{cc}
J_{n, k} & T_{n} \\
T^{k} & J_{k, n}
\end{array}\right)
$$

where $J_{n, k} \in\{0,1\}^{n \times k}$ such that $J_{n, k}(i, j)=1$ for all $i, j ; J_{k, n} \in\{0,1\}^{k \times n}$ such that $J_{k, n}(i, j)=1$ for all $i, j ; T_{n} \in\{0,1\}^{n \times n}$ with $T_{n}(i, j)=1$ if and only if $i \geq j$; and $T^{k} \in\{0,1\}^{k \times k}$ such that $T_{i j}^{k}=1$ if and only if $i \leq j$. For a Vesztergombi permutation select exactly one 1 entry from each row and column. This selection is actually one term in the permanent of the matrix $V_{n+k}$, hence, $\left|\mathcal{V}_{n}^{k}\right|$ is the permanent of the matrix $V_{n+k}$.

For example, for $n=3$ and $k=2$ we have the matrix

$$
V_{3+2}=V_{5}=\left(\begin{array}{ccccc}
1 & 1 & 1 & 0 & 0 \\
1 & 1: 1 & 1 & 0 \\
1 & 1 & 1 & 1 & 1 \\
\hdashline 1 & 1 & 1 & 1 \\
0 & 1 & 1 & 1 & 1
\end{array}\right) \quad \text { and } \operatorname{perm}\left(V_{5}\right)=46
$$

Using other terms, we can say that this is a rook configuration on a board $V_{n+k}$, which is defined as the collection of cells determined by the 1 entries in the matrix.

Next, we recall some general results from rook theory. A rook configuration $\mathcal{A}$ on a binary matrix $A$ are rooks on some of the 1-entries of $A$ such that no two rooks are in the same row or column. The number of cells of $\mathcal{A}$ with no rook weakly to the right in the same row or below in the same column is

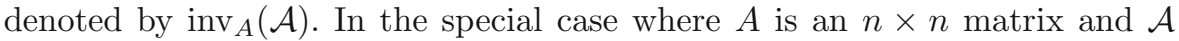
has $n$ rooks, i.e., the configuration corresponds to a permutation, $\pi$, by the representation $\pi_{i}=j, \operatorname{inv}_{A}(\mathcal{A})$ becomes $\operatorname{inv}(\pi)$. The $k$ th $q$-rook number for a board $\mathcal{A}$ is defined by Garsia and Remmel [18] as $R_{k}^{A}(q)=\sum_{\mathcal{A}} q^{\operatorname{inv}_{A}(\mathcal{A})}$, where the sum is over all rook configurations on the matrix $A$ with $k$ rooks.

For example, in Fig. 3 we show the Vesztergombi permutation $\pi=31524 \epsilon$ $V_{3}^{2}$ indicating how to calculate the inversions in this particular case. Notice that the rooks are denoted by black circles and the cells with circles denote the positions counted by the inversion statistic. The number of inversions of this rook configuration is 4 , hence, its weight is $q^{4}$.

It is known [32, Corollary 3] that the $n$th $q$-rook number of the square matrix $J_{n, n}$ is given by $R_{n}^{J_{n, n}}(q)=[n] !_{q}$. Further, Garcia and Remmel [18, p. 


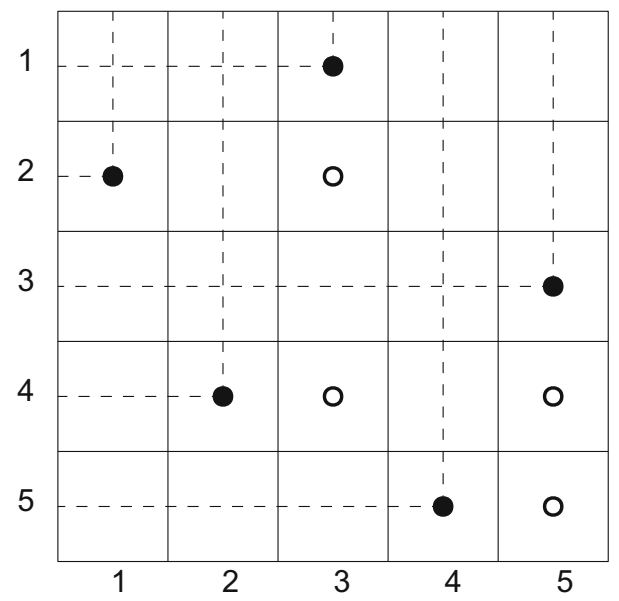

FiguRE 3. Vesztergombi permutation $\pi=31524 \in V_{3}^{2}$

248] showed that the $k$ th $q$-rook number of the $n \times n$ matrix $H_{n}$ with ones on and above the secondary diagonal, that is

$$
\left(H_{n}\right)_{i, j}= \begin{cases}1, & i \leq n-j+1 \\ 0, & \text { otherwise }\end{cases}
$$

is given by $R_{k}^{H_{n}}(q)=q^{\left(\begin{array}{c}n \\ 2\end{array}\right)} S_{n+1, n+1-k}(q)$.

If we denote by $A^{\prime}$ the matrix obtained by reflecting the matrix $A$ upside down, then it is clear that $H_{k}=\left(T_{k}\right)^{\prime}$. Further, from the relation $\operatorname{inv}_{A^{\prime}}(\mathcal{A})=$ $\left(\begin{array}{l}n \\ 2\end{array}\right)-\operatorname{inv}_{A}(\mathcal{A})$ we have that $R_{n}^{A^{\prime}}(q)=q^{\left(\begin{array}{c}n \\ 2\end{array}\right)} R_{n}^{A}(1 / q)$.

Finally, we need the result in [32] about the $(k+n)$ th $q$-rook number of a matrix built from the blocks of matrices $A, B, J_{n, k}$, and $J_{k, n}$ as follows

$$
B / A:=\left(\begin{array}{cc}
B & J_{n, k} \\
J_{k, n} & A
\end{array}\right) .
$$

For this case the $(k+n)$ th $q$-rook number is

$$
R_{k+n}^{B / A}(q)=\sum_{i=0}^{\min (k, n)} R_{k-i}^{A}(q) R_{n-i}^{B^{*}}(q)[i] !_{q} q^{-i^{2}},
$$

where $B^{*}$ is the matrix obtained by rotating the matrix $B$ by 180 degrees.

It is clear that the matrix $V_{n+k}$ is the reflection of the matrix $H_{k}^{*} / H_{n}$. Putting all the results above together, we have

$$
p B_{n, k}(q)=\sum_{\pi \in \mathcal{V}_{n}^{k}} q^{\operatorname{inv}(\pi)}
$$




$$
\begin{aligned}
& =R_{n+k}^{V_{n+k}}(q)=R_{n+k}^{\left(H_{k}^{*} / H_{n}\right)^{\prime}}(q)=q^{\left(\begin{array}{c}
n+k \\
2
\end{array}\right)} R_{n+k}^{H_{k}^{*} / H_{n}}(1 / q) \\
& =q^{\left(\begin{array}{c}
n+k \\
2
\end{array}\right)} \sum_{m=0}^{\min (n, k)} R_{n-m}^{H_{n}}(1 / q) R_{k-m}^{H_{k}}(1 / q)[m] !_{1 / q}^{2} q^{m^{2}} \\
& =q^{\left(\begin{array}{c}
n+k \\
2
\end{array}\right)} \sum_{m=0}^{\min (n, k)} q^{-\left(\begin{array}{c}
n \\
2
\end{array}\right)} S_{n+1, m+1}(1 / q) q^{-\left(\begin{array}{c}
k \\
2
\end{array}\right)} S_{k+1, m+1}(1 / q)[m] !_{1 / q}^{2} q^{m^{2}} \\
& =q^{n k} \sum_{m=0}^{\min (n, k)} S_{n+1, m+1}(1 / q) S_{k+1, m+1}(1 / q)[m] !_{q}^{2} q^{m} .
\end{aligned}
$$

The last equality follows from $[m] !_{1 / q}=[m] !_{q} q^{-\left(\begin{array}{c}m \\ 2\end{array}\right)}$.

Notice that Sjöstrand investigated Coxeter groups in the paper [32]. He obtained the above formula as the Poincaré polynomial of the Bruhat interval [id, w], where $w$ is the maximal element in $A_{n-1}^{S \backslash\left\{s_{k}\right\}}$. That is $p B_{n, k}(q)=$ $\operatorname{Poin}_{[i d, w]}(q)$.

From the definition we have that

$$
\begin{aligned}
& p B_{n, 0}(q)=p B_{0, n}(q)=1, \\
& p B_{n, 1}(q)=p B_{1, n}(q)=(1+q)^{n} .
\end{aligned}
$$

Experimentally, we observed that for $n \geq 2$,

$$
p B_{n, 2}(q)=p B_{2, n}(q)=(1+q) W_{n}(-q),
$$

where $W_{n}(q)$ is the characteristic polynomial of the Sylvester matrix $\mathcal{S}\left(P_{n}(q), P_{n+1}(q)\right)$, with $P_{n}(q):=[n]_{q}=1+q+\cdots+q^{n-1}$.

For example,

$$
\mathcal{S}\left(P_{3}(q), P_{4}(q)\right)=\left(\begin{array}{ccccc}
1 & 1 & 1 & 0 & 0 \\
0 & 1 & 1 & 1 & 0 \\
0 & 0 & 1 & 1 & 1 \\
1 & 1 & 1 & 1 & 0 \\
0 & 1 & 1 & 1 & 1
\end{array}\right)
$$

and its characteristic polynomial is $W_{3}(q)=1-3 q+6 q^{2}-7 q^{3}+5 q^{4}-q^{5}$. Therefore, $p B_{3,2}(q)=p B_{2,3}(q)=(1+q) W_{3}(-q)=1+4 q+9 q^{2}+13 q^{3}+12 q^{4}+6 q^{5}+q^{6}$.

The authors have tried, without success, to establish the above statement.

\section{6. $q$-analogue of Cenkci and Komatsu}

In this section we recall briefly some results of $[15,23]$, where the authors introduced and studied a $q$-analogue of poly-Bernoulli numbers. 
Definition 14. ([15]) Let $n \geq 0$ and $k \geq 1$ be integers and $q$ a real number $(q \neq 0)$. The poly-Bernoulli number with a $q$ parameter is defined by the generating function

$$
\sum_{n=0}^{\infty} B_{n, q}^{(k)} \frac{t^{n}}{n !}=\frac{q \operatorname{Li}_{k}\left(\frac{1-e^{-q t}}{q}\right)}{1-e^{-q t}} .
$$

The authors have derived the analogues of the formulas of type (2) and (4). For example, we have

$$
B_{n, q}^{(k)}=\sum_{m=0}^{n}\left\{\begin{array}{l}
n \\
m
\end{array}\right\} \frac{(-q)^{n-m} m !}{(m+1)^{k}} .
$$

Further, the analogue of (3) is true for all negative integers $k$ :

$$
B_{n, q}^{(k)}=q \sum_{j=0}^{\min (n, k)}(j !)^{2} S_{2}(n, j, q) S_{2}^{q^{-1}}(k+1, j+1),
$$

where

$$
\begin{aligned}
S_{2}(n, j, q) & =\sum_{k=0}^{n}\left(\begin{array}{l}
n \\
k
\end{array}\right) q^{n-k}\left\{\begin{array}{l}
k \\
j
\end{array}\right\} \text { and } \\
\sum_{n=j}^{\infty} S_{2}^{q^{-1}}(n+1, j+1) \frac{t^{n}}{n !} & =\frac{\left(q^{-1} e^{t}-1\right)^{j} q^{-1} e^{t}}{j !} .
\end{aligned}
$$

We point out the similarity of the recursion (4) and the recursion that was derived for the so defined poly-Bernoulli numbers with a $q$ parameter in [15]. We write the formula in the form that emphasizes this similarity better $(k \geq 1)$.

$$
B_{n, q}^{-(k+1)}=(n+1) B_{n, q}^{(-k)}+\sum_{i=1}^{n-1} q^{i}\left(\begin{array}{c}
n \\
i+1
\end{array}\right) B_{n-i, q}^{(-k)} .
$$

For the original formula (4) $\Gamma$-free matrices give a transparent explanation. $\Gamma$-free matrices were introduced in [8] as 01 matrices avoiding the following two $2 \times 2$ submatrices

$$
\Gamma=\left\{\left(\begin{array}{ll}
1 & 1 \\
1 & 0
\end{array}\right),\left(\begin{array}{ll}
1 & 1 \\
1 & 1
\end{array}\right)\right\} .
$$

For example, the $46 \Gamma$-free matrices of size $2 \times 3$ appear in Fig. 4 . Note that we use black squares for the ones and white squares for the zeros.

A bijective proof of the fact that $n \times k \Gamma$-free matrices are enumerated by the poly-Bernoulli numbers $B_{n}^{(-k)}$ was given in [8] and [9]. In [8] the authors give the explanation of the recursion (4).

We use now this observation in order to define a combinatorial interpretation of the polynomials $B_{n, q}^{(-k)}$, with $k \geq 1$. We introduce two notation. We call a 1 entry in a matrix corner 1 if there is at least 1 entry below it in the 

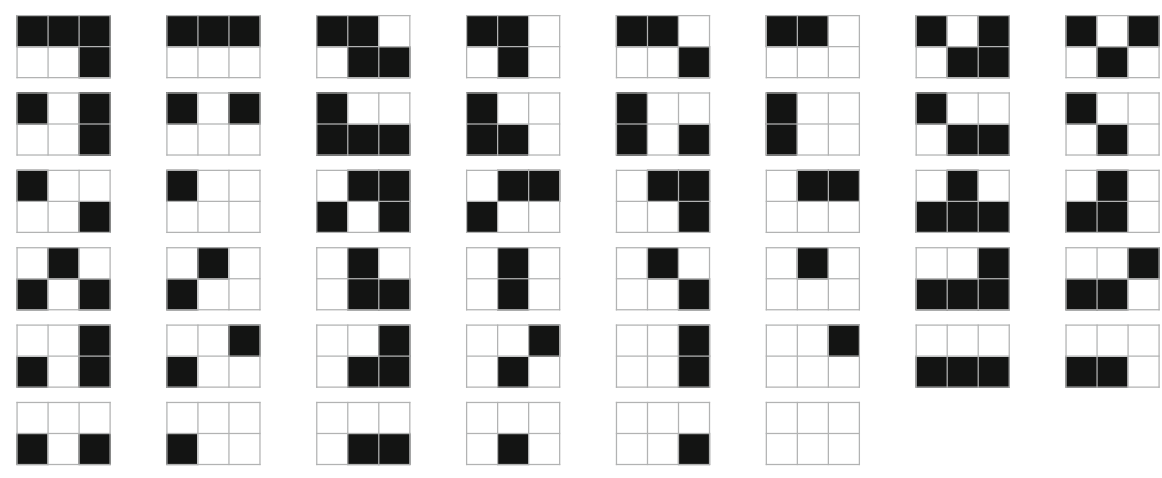

Figure 4 . $\Gamma$-free matrices of size $2 \times 3$

same column and only zeros to its right in the same row. Note that we require to have at least one 0 to the right, so a corner 1 does not appear in the last column. Further, we define the height of a $\Gamma$-free matrix as the number of rows that do not contain any corner 1 . Given a matrix $M$, we let $c(M)$ denote the number of corner 1's in the matrix $M$ and $h(M)$ the height of the matrix $M$. For example, for the $\Gamma$-free matrix

$$
M=\left(\begin{array}{lll}
0 & \mathbf{1} & 0 \\
0 & 0 & 1 \\
0 & \mathbf{1} & 0 \\
1 & 1 & 0
\end{array}\right),
$$

we have that $c(M)=2$ and $h(M)=2$. As next, we define to each height, $h$, the polynomial $p_{h}$ as the $q$-poly-Bernoulli number at the parameter -1 . That is, $p_{h}=B_{h, q}^{(-1)}$. So for instance,

$$
\begin{aligned}
& p_{1}=2, \\
& p_{2}=-2 q+6 \\
& p_{3}=2 q^{2}-18 q+24 \\
& p_{4}=-2 q^{3}+42 q^{2}-144 q+120, \\
& p_{5}=2 q^{4}-90 q^{3}+600 q^{2}-1200 q+720 .
\end{aligned}
$$

We can view these polynomials also as the weight associated to the set of 01 vectors of length $h$. We define the following weight to a given $\Gamma$-free matrix, $M$ :

$$
w(M)=(-1)^{c(M)} q^{c(M)} p_{h(M)} .
$$

For example, for the matrix $M$ in (13), we have that

$$
w(M)=q^{2} p_{2}=q^{2}(-2 q+6)=-2 q^{3}+6 q^{2} .
$$


The main result of this section is the following theorem.

Theorem 15. We have

$$
B_{n, q}^{(-k)}=\sum_{M \in \mathcal{G}(n,|k|)} w(M)=\sum_{M \in \mathcal{G}(n,|k|)}(-1)^{c(M)} q^{c(M)} p_{h(M)},
$$

where $\mathcal{G}(n, k)$ is the set of $\Gamma$-free matrices with $n$ rows and $k$ columns.

Proof. One of the crucial properties of a $\Gamma$-free matrix is that if a column contains at least two 1s, the elements to the right of the 1s (except the lowest 1 in the column) are all 0s. Hence, all the 1s in such a column are corner 1s except the lowest 1 . This property allows to build a $\Gamma$-free matrix as follows: choose some row indices, $\mathcal{R}$, write into these rows in the first column a 1 , and fill the rest of these rows with $0 \mathrm{~s}$, except the bottom most row, the greatest element in $\mathcal{R}$. For the rest of the matrix there are no further restrictions, it can be constructed as an arbitrary $\Gamma$-free matrix with $n-|\mathcal{R}|+1$ rows and $k$ columns. This construction is given in the recurrence (4). The recurrence (12) is the version of (4) with respect to the weight defined above. Namely, assume first, that the first column doesn't contain any corner 1s. There are $n+1$ possible such 01 columns, the all-0 column, and those that contain only one 1 entry. Such a column doesn't influence the rest of the matrix and doesn't add any factor to the weight (except maybe in determining the height of the matrix). Assume now that the first column contains $i$ corner 1s. On one hand, we know then that in these $i$ rows there are only 0 s in the remaining columns, so we can ignore these rows. On the other hand, we keep track of these corner 1s (and rows) by adding a $q^{i}$ factor to the weight of the matrix. There are $\left(\begin{array}{c}n \\ i+1\end{array}\right)$ possibilities to choose the $i$ corner 1 s and a 1 as the lowest 1 in the first column. After iterating this process, i.e., deleting the rows of corner 1s, a matrix without any corner 1 is left, which has a weight depending only on the number of its rows. (Note that each row contains at least 1 corner 1s, so the height of the matrix is the number of rows of the matrix reduced by the number of corner 1s.) As the outcome of this process a certain matrix obtains exactly the weight $w(M)$ we defined above and we have the recurrence (12).

As next we give a different combinatorial interpretation of $B_{n, q}^{(-k)}$ based on the formula (10) and using lonesum matrices. For this sake, first we introduce colored lonesum matrices. Because of the special features of the lonesum matrix the set of columns that can arise in a matrix simultaneously has certain conditions. As we mentioned earlier, two submatrices are forbidden in a lonesum matrix, which has the consequence, that any two columns differ only at some entries, having one column at these places 1 while the other 0 , but all the other entries are identical. We determine sets of columns that can arise simultaneously in a lonesum matrix, i.e., sets of 01 columns of a given length $n$, with the above property. We call such a set lonesum alphabet. We also require 
that a lonesum alphabet contains the "least" and "greatest" column, the all-0 and all- 1 column. For instance, for $n=2$ there are three lonesum alphabets:

$$
\left\{\overline{\left(\begin{array}{l}
0 \\
0
\end{array}\right)} \overline{\left(\begin{array}{l}
1 \\
0
\end{array}\right)} \overline{\left(\begin{array}{l}
1 \\
1
\end{array}\right)}\right\}\left\{\overline{\overline{\left(\begin{array}{l}
0 \\
0
\end{array}\right)}} \overline{\overline{\left(\begin{array}{l}
0 \\
1
\end{array}\right)}} \overline{\overline{\left(\begin{array}{l}
1 \\
1
\end{array}\right)}}\right\}\left\{\overline{\overline{\left(\begin{array}{l}
0 \\
0
\end{array}\right)}} \overline{\overline{\left(\begin{array}{l}
1 \\
1
\end{array}\right)}}\right\}
$$

On the other hand, for example, the lonesum matrix $E$

$$
E=\left(\begin{array}{lll}
1 & 1 & 1 \\
0 & 1 & 1 \\
0 & 0 & 1 \\
0 & 1 & 1
\end{array}\right)
$$

can be built from the following lonesum alphabets

$$
\begin{gathered}
\left\{\left(\begin{array}{l}
0 \\
0 \\
0 \\
0
\end{array}\right)\left(\begin{array}{l}
1 \\
0 \\
0 \\
0
\end{array}\right)\left(\begin{array}{l}
1 \\
1 \\
0 \\
1
\end{array}\right)\left(\begin{array}{l}
1 \\
1 \\
1 \\
1
\end{array}\right)\right\}, \quad\left\{\left(\begin{array}{l}
0 \\
0 \\
0 \\
0
\end{array}\right)\left(\begin{array}{l}
1 \\
0 \\
0 \\
0
\end{array}\right)\left(\begin{array}{l}
1 \\
1 \\
0 \\
0
\end{array}\right)\left(\begin{array}{l}
1 \\
1 \\
0 \\
1
\end{array}\right)\left(\begin{array}{l}
1 \\
1 \\
1 \\
1
\end{array}\right)\right\}, \\
\left\{\left(\begin{array}{l}
0 \\
0 \\
0 \\
0
\end{array}\right)\left(\begin{array}{l}
1 \\
0 \\
0 \\
0
\end{array}\right)\left(\begin{array}{l}
1 \\
0 \\
0 \\
1
\end{array}\right)\left(\begin{array}{l}
1 \\
1 \\
0 \\
1
\end{array}\right)\left(\begin{array}{l}
1 \\
1 \\
1 \\
1
\end{array}\right)\right\}
\end{gathered}
$$

We associate a color to each alphabet. We build from each alphabet every possible matrix and color the matrix with the color of the alphabet indicating (as a reminder) from which alphabet it is originated. In this way, we obtain colored lonesum matrices. Clearly, there are several copies of certain lonesum matrices in different colors. For instance, the all-0 matrix is a lonesum matrix, that will appear in every possible color. We associate to each colored lonesum matrix $M$ that has the color of an alphabet of size $m+1$ the weight $(-q)^{n-m}$, and denote this weight by $w^{+}(M)$. For instance, the $2 \times 1$ colored lonesum matrices with weight 1 are

$$
\overline{\left(\begin{array}{l}
0 \\
0
\end{array}\right)}, \overline{\overline{\left(\begin{array}{l}
0 \\
0
\end{array}\right)}}, \overline{\left(\begin{array}{l}
1 \\
1
\end{array}\right)}, \overline{\overline{\left(\begin{array}{l}
1 \\
1
\end{array}\right)}}, \overline{\left(\begin{array}{l}
1 \\
0
\end{array}\right)}, \overline{\overline{\left(\begin{array}{l}
0 \\
1
\end{array}\right)}}
$$

And with weight $-q$ :

$$
\overline{\overline{\left(\begin{array}{l}
0 \\
0
\end{array}\right)}} \overline{\overline{\overline{\left(\begin{array}{l}
1 \\
1
\end{array}\right)}}}
$$

We claim the following statement.

Theorem 16. We have

$$
B_{n, q}^{(-k)}=\sum_{M \in \mathcal{C} \mathcal{L}(n, k)} w^{+}(M),
$$


where $\mathcal{C} \mathcal{L}(n, k)$ denotes the set of colored lonesum matrices with $n$ rows and $k$ columns.

Proof. Clearly, there are $(m+1)^{k}$ lonesum matrices with $n$ rows and $k$ columns that are built from a lonesum alphabet containing $m+1$ columns. The number of alphabets with $m+1$ columns is easy to determine. The all- 0 column is surely in the alphabet, for the next column choose some 0 entries to flip into 1 , for the next column choose again some more 0s to flip into 1, and so on, until we arrive at the all-1 column. This process actually indicates an ordered partition of $n$ into $m$ blocks. So there are $m !\left\{\begin{array}{c}n \\ m\end{array}\right\}$ lonesum alphabets with $m+1$ columns. The formula (10) follows and hence the theorem is proven.

Note that setting $q=1$, we have (2). Based on this previous construction we can associate weights directly to lonesum matrices. Consider a lonesum matrix $M$. Associate to $M$ the sum of the weights of all its colored copies of it. Let $w^{++}$denote this weight. For instance,

$$
\begin{array}{r}
w^{++}\left(\begin{array}{l}
0 \\
0
\end{array}\right)=-q+2, \quad w^{++}\left(\begin{array}{l}
1 \\
1
\end{array}\right)=-q+2, \quad w^{++}\left(\begin{array}{l}
0 \\
0
\end{array}\right)=1, \\
w^{++}\left(\begin{array}{l}
0 \\
0
\end{array}\right)=1 .
\end{array}
$$

Or the weight of the matrix $E: w^{++}(E)=-q+2$, while it can be built from a lonesum alphabet of size 4 and from two different lonesum alphabets of size 5. Each matrix has a weight depending on how many different columns it contains. If the matrix has $c_{1}, \ldots, c_{\ell}$ different columns (all-0 and all-1 different columns) the weight of the matrix is

$$
w^{++}\left(M_{\underline{c}}\right)=\sum_{i=0}^{\min (k-\ell, n-\ell-1)}(-1)^{\ell+i} v(\underline{c}, i) q^{i},
$$

where $v(\underline{c}, i)$ is the number of ways the column set $\underline{c}$ can be augmented by $i$ further columns to create a lonesum alphabet (beyond the all-0 and all-1 columns). Then we have

Theorem 17. We have

$$
B_{n, q}^{(-k)}=\sum_{M \in \mathcal{L}(n, k)} w^{++}(M),
$$

where $\mathcal{L}(n, k)$ denotes the set of lonesum matrices with $n$ rows and $k$ columns.

Proof. The statement is clear from the previous discussion.

For instance, the matrices with $n$ rows that contain only all-0 or all-1 (or both) has the weight $B_{n, q}^{0}$. In this way we have given a combinatorial meaning of the polynomials also for the case of $k=0$. 
Remark 18. Some special settings of $q$ have combinatorial importance. For instance, $B_{n, 1-q}^{(0)}$ are the Eulerian polynomials, $A_{n}(q) .(-1)^{n+1} B_{2 n+1,2}^{(0)}$ are the tangent numbers (see sequence A000182 [33]) that count alternating permutations and $2^{n} B_{n, \frac{1}{2}}^{(1)}$ (see sequence A000629 in [33]) is the number of cyclically ordered partitions of $n+1$ labeled points.

\section{Conclusion}

In this work we presented some possible variants of $q$-poly-Bernoulli numbers. These enumerations are heavily based on the combinatorics of poly-Bernoulli numbers, i.e., the weight $q$ measures some of the parameters that arise naturally in combinatorial objects enumerated by the poly-Bernoulli numbers. However, as we mentioned several generalizations can be found in the literature that are motivated analytically from a number theoretic point of view. We provided a combinatorial interpretation for one such generalization in the last section. We would like to recall two interesting examples - though there are several others - in order to motivate the reader to continue the combinatorial study of this line of research.

Komatsu [23] defined the q-poly-Bernoulli polynomials with parameter $\rho$, $B_{n, \rho, q}^{(k)}$ for $n, k$ integers, and $\rho, q$ real numbers with $\rho \neq 0$ and $0 \leq q<1$ as follows:

$$
\sum_{n=0}^{\infty} B_{n, \rho, q}^{(k)}(z) \frac{t^{n}}{n !}=\frac{\rho}{1-e^{-\rho t}} \operatorname{Li}_{k, q}\left(\frac{1-e^{-\rho t}}{\rho}\right) e^{-t z},
$$

where $\operatorname{Li}_{k, q}(z)$ is the $q$-polylogarithm function defined by $\operatorname{Li}_{k, q}(z)=\sum_{n=1}^{\infty} t^{n} /$ $[n]_{q}^{k}$. It holds that $\lim _{q \rightarrow 1} B_{n, \rho, q}^{k}(z)=B_{n, \rho}^{(k)}(z)$. In particular, for $z=0$, $\lim _{q \rightarrow 1} B_{n, \rho, q}^{k}(0)=B_{n, \rho}^{(k)}(0)$, the poly-Bernoulli numbers with parameter $\rho$. The question naturally arises whether the combinatorial interpretation via $\Gamma$ free matrices could be extended to an interpretation of the $q$-poly-Bernoulli polynomials with parameter $\rho$.

Moreover, Komatsu and Szalay [26] extended this notion even further and introduced q-multiparameter-poly-Bernoulli polynomials as

$$
B_{n, L, A, q}^{(k)}(z)=\sum_{m=0}^{n} S_{2}(n, m, A) m ! \sum_{i=0}^{m}\left(\begin{array}{c}
m \\
i
\end{array}\right) \frac{(-z)^{i} \ell^{m-i+1}}{[m-i+1]_{q}^{k}},
$$

where $S_{2}(n, m, A)$ denotes the multiparameter Stirling number of second kind defined for an $n$-tuple of real numbers $A=\left(\alpha_{0}, \alpha_{1}, \ldots, \alpha_{n-1}\right)$ by the equality

$$
\sum_{m=0}^{n} S_{2}(n, m, A)\left(t-\alpha_{0}\right)\left(t-\alpha_{1}\right) \cdots\left(t-\alpha_{m-1}\right)=t^{n} .
$$

In $[26]$ we find several identities involving these polynomials. 


\section{Acknowledgements}

The authors would like to thank the anonymous referee for several useful comments. The second author was partially supported by Universidad Nacional de Colombia, Project No. 46240.

Funding Open access funding provided by University of Public Service.

Open Access. This article is licensed under a Creative Commons Attribution 4.0 International License, which permits use, sharing, adaptation, distribution and reproduction in any medium or format, as long as you give appropriate credit to the original author(s) and the source, provide a link to the Creative Commons licence, and indicate if changes were made. The images or other third party material in this article are included in the article's Creative Commons licence, unless indicated otherwise in a credit line to the material. If material is not included in the article's Creative Commons licence and your intended use is not permitted by statutory regulation or exceeds the permitted use, you will need to obtain permission directly from the copyright holder. To view a copy of this licence, visit http:// creativecommons.org/licenses/by/4.0/.

Publisher's Note Springer Nature remains neutral with regard to jurisdictional claims in published maps and institutional affiliations.

\section{References}

[1] Andrews, G.E., Askey, R., Roy, R.: Special Functions. In: Encyclopedia of Mathematics and its Applications, vol. 71. Cambridge University Press (1999)

[2] Arakawa, T., Kaneko, M.: Multiple zeta values, poly-Bernoulli numbers and related zeta functions. Nagoya Math. J. 153, 189-209 (1999)

[3] Arakawa, T., Kaneko, M.: On poly-Bernoulli numbers. Comment. Math. Univ. St. Pauli 48, 159-167 (1999)

[4] Bayad, A., Hamahata, Y.: Arakawa-Kaneko $L$-functions and generalized poly-Bernoulli polynomials. J. Number Theory 131, 1020-1036 (2011)

[5] Bayad, A., Hamahata, Y.: Multiple polylogarithms and multi-poly-Bernoulli polynomials. Funct. Approx. Comment. Math. 46, 45-61 (2012)

[6] Bényi, B.: Advances in bijective combinatorics. PhD thesis, (2014). available at http:// www.math.u-szeged.hu/phd/dreposit/phdtheses/benyi-beata-d.pdf

[7] Bényi, B., Hajnal, P.: Combinatorics of poly-Bernoulli numbers. Studia Sci. Math. Hungarica 52, 537-558 (2015)

[8] Bényi, B., Hajnal, P.: Combinatorial properties of poly-Bernoulli relatives. Integers 17(A31), 1-26 (2017)

[9] Bényi, B., Nagy, G.V.: Bijective enumerations of $\Gamma$-free matrices. Adv. Appl. Math. 96, 195-215 (2018)

[10] Bényi, B., Méndez, M., Ramírez, J.L., Wakhare, T.: Restricted $r$-Stirling numbers and their combinatorial applications. Appl. Math. Comput. 348, 186-205 (2019)

[11] Bényi, B., Ramírez, J.L.: Some applications of $S$-restricted set partitions. Period. Math. Hungar. 78, 110-127 (2019)

[12] Brewbaker, C.: A combinatorial interpretation of the poly-Bernoulli numbers and two Fermat analogues. Integers 8, 1-9 (2008) 
[13] Bukata, M., Kulwicki, R., Lewandowski, N., Pudwell, L., Roth, J., Wheeland, T.: Distributions of statistics over pattern-avoiding permutations. J. Integer Seq. 3, Article 19.2.6 (2019)

[14] Carlitz, L.C.: q-Bernoulli numbers and polynomials. Duke Math. J. 15, 987-1000 (1948)

[15] Cenkci, M., Komatsu, T.: Poly-Bernoulli numbers and polynomials with a $q$-parameter. J. Number Theory 152, 38-54 (2015)

[16] Cigler, J.: A new q-analogue of Stirling numbers. Sitzunber. Abt. II(201), 97-109 (1992)

[17] Corcino, R.B., Fernandez, J.C.: A combinatorial approach for $q$-analogue of $r$-Stirling numbers. British J. Math. Comput. Sci. 4, 1268-1279 (2014)

[18] Garsia, A.M., Remmel, J.B.: Q-counting rook configurations and a formula of Frobenius. J. Combin. Theory Ser. A 41, 246-275 (1986)

[19] Jackson, F.H.: On q-definite integrals. Quart. J. 41, 193-203 (1910)

[20] Kaneko, M.: Poly-Bernoulli numbers. J. Théor. Nombres Bordeaux 9, 221-228 (1997)

[21] Kaneko, M.: Multiple Zeta Values and Poly-Bernoulli Numbers. Tokyo Metropolitan University Seminar Report (1997)

[22] Komatsu, T.: Poly-Cauchy numbers. Kyushu J. Math. 67, 143-153 (2013)

[23] Komatsu, T.: q-poly-Bernoulli numbers and $q$-poly-Cauchy numbers with a parameter by Jackson's integrals. Indag. Math. 27(1), 100-111 (2016)

[24] Komatsu, T., Liptai, K., Mező, I.: Incomplete poly-Bernoulli numbers associated with incomplete Stirling numbers. Publ. Math. Debrecen 88, 357-368 (2016)

[25] Komatsu, T., Ramírez, J.L.: Generalized poly-Cauchy and poly-Bernoulli numbers by using incomplete $r$-Stirling numbers. Aequat. Math. 91, 1055-1071 (2017)

[26] Komatsu, T., Szalay, L.: $q$-multiparameter-Bernoulli polynomials and $q$-multiparameterCauchy polynomials by Jackson's integrals. Integers 16(A39), 1-11 (2016)

[27] Kim, H.K., Krotov, D.S., Lee, J.Y.: Matrices uniquely determined by their lonesums. Linear Algebra Appl. 438, 3107-3123 (2013)

[28] Launois, S.: Combinatorics of $\mathcal{H}$-primes in quantum matrices. J. Algebra 309, 139-167 (2007)

[29] Mansour, T.: Combinatorics of Set Partitions. CRC Press, Boca Raton (2012)

[30] Mansour, T., Schork, M.: Commutations Relations, Normal Ordering, and Stirling Numbers. CRC Press, Boca Raton (2015)

[31] Ohno, Y., Sasaki, Y.: On poly-Euler numbers. J. Aust. Math. Soc. 103, 126-144 (2017)

[32] Sjöstrand, J.: Bruhat intervals as rooks on skew Ferrers boards. J. Combin. Theory Ser. A 114, 1182-1198 (2007)

[33] Sloane, N.J.A.: The On-Line Encyclopedia of Integer Sequences, http://oeis.org

[34] Son, J.-W., Kim, M.-S.: On poly-Eulerian numbers. Bull. Korean Math. Soc. 36, 47-61 (1999)

[35] Vesztergombi, K.: Permutations with restriction of middle strength. Studia Sci. Math. Hungarica 9, 181-185 (1974)

Beáta Bényi

Faculty of Water Sciences

University of Public Service

Baja

Hungary

e-mail: benyi.beata@uni-nke.hu

José L. Ramírez

Departamento de Matemáticas

Universidad Nacional de Colombia

Bogotá

Colombia

e-mail: jlramirezr@unal.edu.co

Received: January 9, 2021

Revised: June 28, 2021

Accepted: June 30, 2021 